\title{
Laser polarization dependence of strong-field ionization in lithium niobate
}

\author{
Vincent Wanie $\odot,{ }^{1,2,3, *}$ Tian-Jiao Shao $\odot,{ }^{4,5}$ Philippe Lassonde, ${ }^{1}$ Francesca Calegari, ${ }^{2,3,6}$ François Vidal $\odot,{ }^{1}$ Heide Ibrahim $\odot,{ }^{1}$ \\ Xue-Bin Bian $\odot,{ }^{4, \dagger}$ and François Légaré ${ }^{1, \ddagger}$ \\ ${ }^{1}$ Centre Énergie Matériaux Télécommunications, Institut National de la Recherche Scientifique, Varennes, Québec J3X 1S2, Canada \\ ${ }^{2}$ Center for Free-Electron Laser Science, DESY, Hamburg 22607, Germany \\ ${ }^{3}$ Institute for Photonics and Nanotechnologies CNR-IFN, Milano 20133, Italy \\ ${ }^{4}$ State Key Laboratory of Magnetic Resonance and Atomic and Molecular Physics, Wuhan Institute of Physics and Mathematics, Innovation \\ Academy for Precision Measurement Science and Technology, Chinese Academy of Sciences, Wuhan 430071, China \\ ${ }^{5}$ School of Physics, University of Chinese Academy of Sciences, Beijing 100049, China \\ ${ }^{6}$ Physics Department, Universität Hamburg, Hamburg 22761, Germany
}

(Received 6 April 2020; revised manuscript received 8 June 2020; accepted 9 June 2020; published 26 June 2020)

\begin{abstract}
Laser micromachining techniques using femtosecond laser pulses are employed for the fabrication of a wide range of devices, from optical waveguides and fiber Bragg gratings to microfluidic systems. In this perspective, it is important to characterize how transparent materials respond to the high-intensity laser field in order to fully understand and control the strong-field ionization process at the core of such techniques. Here, we characterize the laser polarization dependence of $y$-cut and $x$-cut ferroelectric lithium niobate $\left(\mathrm{LiNbO}_{3}\right)$ upon ionization. Using linearly polarized 1800 -nm femtosecond pulses, we perform single-shot laser-induced ablation measurements to obtain a macroscopic observable with a large contrast compared to all-optical techniques. In the tunnel ionization regime, the crystal orientation can be correlated with the structural symmetry/asymmetry of the material. This is revealed through a variation of the ablated area when the laser polarization is rotated with respect to the $c$ axis of the crystal. It is further found that ablation is more pronounced when the laser polarization is oriented towards angular regions containing $\mathrm{Nb}-\mathrm{O}$ bonds of the unit cell, identifying the main chemical bonds contributing to the ionization of the material. The experimental results are supported by numerical simulations based on a two-band model for $\mathrm{LiNbO}_{3}$.
\end{abstract}

DOI: 10.1103/PhysRevB.101.214311

\section{INTRODUCTION}

Laser micromachining techniques such as femtosecond nonlinear lithography and laser-induced ablation (LIA) have proven to be of great use for high-resolution material processing [1] and are employed for a variety of applications including the fabrication of microfluidic devices and photonic components [2,3]. Particularly in photonics, lithium niobate $\left(\mathrm{LiNbO}_{3}\right)$ is an attractive material due to its strong piezoelectric, electro-optic, acousto-optic, and nonlinear properties. Indeed, the combination of such properties within the same substrate makes it very convenient, as it can fulfill several functions in the realization of integrated optical devices. For instance, recent efforts have been developed to produce high-efficiency waveguide amplifiers/lasers through doped $\mathrm{LiNbO}_{3}[4,5]$. In addition, the fabrication of $\mathrm{Y}$-branch optical waveguides in $\mathrm{LiNbO}_{3}$, based solely on laser micromachining, has also been demonstrated for applications in the midinfrared spectral range $(4 \mu \mathrm{m})$ [6]. In this case, the laser-ablated regions were created by carefully choosing the femtosecond pulse parameters. In fact, the level of precision

\footnotetext{
*vincent.wanie@desy.de

†xuebin.bian@wipm.ac.cn

\#legare@emt.inrs.ca
}

achieved for a specific material is limited by our understanding of the interaction between the strong laser field and the sample that undergoes ionization [7]. On the one hand, this interaction critically depends on the material's properties. The role of the spontaneous polarization in the strong-field ionization of ferroelectric $\mathrm{LiNbO}_{3}$ was recently highlighted through LIA measurements [8]. On the other hand, laser pulse characteristics such as the intensity, duration, wavelength, spatial energy distribution, and polarization state are of significant importance [9-13]. Careful characterization of these parameters is thus necessary to enhance the precision of laser micromachining techniques.

For linearly polarized femtosecond pulses, in particular, the orientation of the polarization plane with respect to the crystal axes has shown peculiar effects. From LIA measurements, Li et al. reported how the incident polarization angle affects the ionization of cubic crystals with increasing bandgap energy [13]. At proper fluences, chosen to minimize the contribution of impact ionization [14], the fourfold rotational symmetry of the samples was reflected in the modulation of the ablated area and interpreted as a result of an orientation-dependent effective electron mass $m_{e}$. Based on a similar interpretation, transmission measurements by Gertsvolf et al. [12] and Golin et al. [15] have also demonstrated the critical role of $m_{e}$ for the generation of free carriers in the conduction band of materials. In the aforementioned works, the crystal was always oriented 
to reveal the axis of highest symmetry ( $n$-fold rotation), i.e., the $c$ axis $\left(z\right.$ cut) for $\mathrm{LiNbO}_{3}$ crystals. Other crystal orientations can exhibit more subtle symmetry features that can be difficult to observe. The LIA technique chosen here, together with our theoretical support, is, however, capable of addressing these subtle changes due to crystal symmetries.

In this article, we study the strong-field ionization response of $y$-cut and $x$-cut lithium niobate $\left(\mathrm{LiNbO}_{3}\right)$ to femtosecond laser pulses when rotating the incident linear polarization. We use LIA in the tunneling regime to identify characteristic features for each crystal cut, notably the absence or presence of a mirror symmetry within the crystal plane probed by the laser field. Moreover, ablation is found to be most efficient for orientations where the laser polarization is within those angular regions containing the $\mathrm{Nb}-\mathrm{O}$ bonds of the unit cell. First-principles calculations and numerical simulations based on a two-band model show how the Nb-O bonds contribute almost exclusively to the band structure of the material and to its conduction-band population during the interaction with the laser field.

\section{EXPERIMENTS}

For the experiments at the ALLS infrastructure (Canada), a white-light-seeded optical parametric amplifier (TOPAS; light conversion) was pumped with a $2.5 \mathrm{kHz}$ titanium-doped sapphire (KMLabs) laser and delivered 70-fs pulses centered at $1800 \mathrm{~nm}(0.69 \mathrm{eV})$. To ensure that tunneling is the main ionization channel during the LIA process, this photon energy was chosen as it is well below the experimentally obtained 3.78-eV bandgap energy for $\mathrm{LiNbO}_{3}$ [16]. Higher-bandgap materials such as quartz $(\sim 9 \mathrm{eV})$ can be studied with conventional $800-\mathrm{nm}(1.55-\mathrm{eV})$ laser sources. The pulse duration is another critical parameter that determines the ablation regime. While the use of picosecond pulses can alter the reproducibility of ablation due to significant heating effects of the crystal lattice, those are minimized with laser pulses of a few tens of femtoseconds and hence lead to a higher level of precision $[7,17]$. The beam was spatially filtered and focused with an $f=50 \mathrm{~mm}$ off-axis parabolic mirror. Its diameter at focus $\left(1 / e^{2}\right.$ of intensity) was evaluated to $39 \mu \mathrm{m}$ from the fits of images acquired with a CCD camera. Single-shot exposition onto the sample surface was achieved using a fast ceramic motor translation stage (Nanomotion Ltd.) moving in the direction parallel to the crystal surface. An imaging system exploiting the Fresnel back reflection from the sample surface was built and employed to precisely adjust the crystal surface position at the laser focus and the surface parallelism with respect to the translation axis. After the ablation series was completed, images of the ablated craters were acquired using an optical microscope (Nikon Eclipse) with a $100 \times$ objective. Their area was evaluated by a fitting routine and the standard deviation was defined over 10 single-shot ablated craters per orientation. A sketch of the relevant crystal planes is shown in Fig. 1(a). The linear polarization of the laser pulse is rotated at several incident angles $\theta$ relative to the $c$ axis of $\mathrm{LiNbO}_{3}$ and the beam is focused onto the surface to ablate the material. The fluence is set just above the threshold for ablation and the corresponding intensity, taking into account reflective losses, ensures that ionization is described by tunneling with the (a)
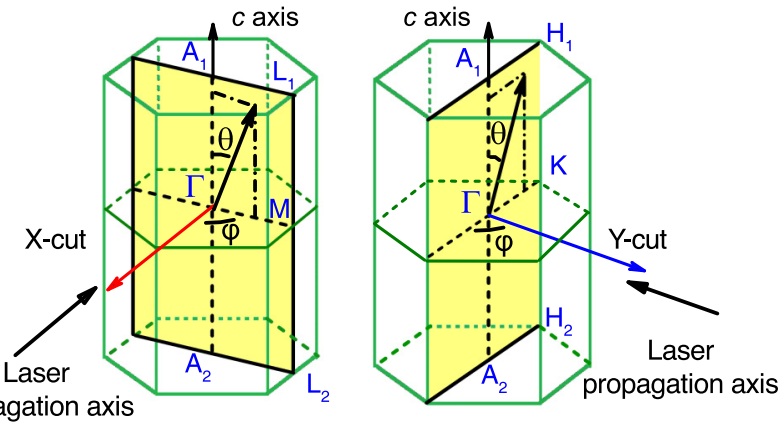

(b)

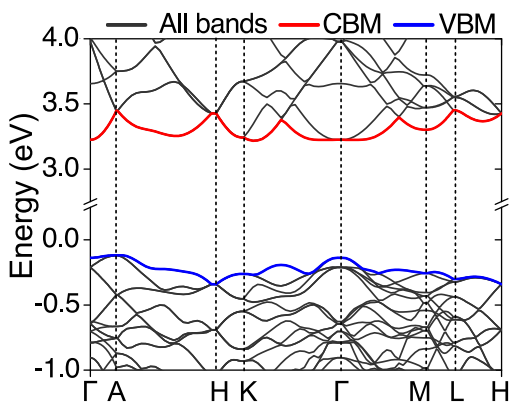

FIG. 1. (a) The laser polarization is rotated by the angle $\theta$ with respect to the $c$ axis of $\mathrm{LiNbO}_{3}$ for ablating both $x$-cut (left) and $y$-cut (right) samples with an intensity of $2.3 \times 10^{13} \mathrm{~W} / \mathrm{cm}^{2}$. Shown are the $\Gamma-\mathrm{A}-\mathrm{L}-\mathrm{M}$ and $\Gamma-\mathrm{A}-\mathrm{H}-\mathrm{K}$ planes of the Brillouin zone, corresponding, respectively, to the $x$ cut and $y$ cut. (b) DFT-calculated band structure with valence-band maximum (VBM; blue curve) and conduction-band minimum (CBM; red curve).

Keldysh parameter $\gamma=0.47$. An angular scan is performed for $y$-cut and $x$-cut crystals, corresponding, respectively, to the $\Gamma-\mathrm{A}-\mathrm{H}-\mathrm{K}$ and $\Gamma-\mathrm{A}-\mathrm{L}-\mathrm{M}$ planes of the Brillouin zone. The band structure of $\mathrm{LiNbO}_{3}$ is shown in Fig. 1(b).

\section{RESULTS AND DISCUSSION}

\section{A. Angle-resolved LIA measurements}

The experimental ablation results are presented in Fig. 2, showing the ablated area of the material as a function of the laser polarization angle $\theta$. We first consider the most symmetric case in Fig. 2(a), corresponding to the $y$-cut crystal ablated at an intensity of $2.3 \times 10^{13} \mathrm{~W} / \mathrm{cm}^{2}$. From 0 to $360^{\circ}$ the ablation pattern shows a symmetric behavior with respect to $180^{\circ}$, identified by the vertical dashed blue line and delimiting the center of the angular scan. Furthermore, each half of the scan shows a characteristic minimum at about $90^{\circ}\left(270^{\circ}\right)$ indicated by the vertical dashed yellow lines. Also shown are two symmetrically distributed maxima, at $40^{\circ}\left(220^{\circ}\right)$ and $140^{\circ}$ $\left(320^{\circ}\right)$, i.e., $50^{\circ}$ away from the minimum.

In Fig. 2(b), the orange squares show the polarization dependence of the $x$-cut crystal for the same laser intensity. In this case, an asymmetric structure is observed with respect to a sharp and reproducible minimum at about $90^{\circ}$. In its vicinity, the ablated area drops abruptly on the left-hand side (shaded area) and rises more smoothly on the right-hand side. This asymmetric structure is also reproduced when the laser intensity is reduced to $1.9 \times 10^{13} \mathrm{~W} / \mathrm{cm}^{2}$ (black triangles). For visualization, the initial angular range from $-15^{\circ}$ to $195^{\circ}$ 

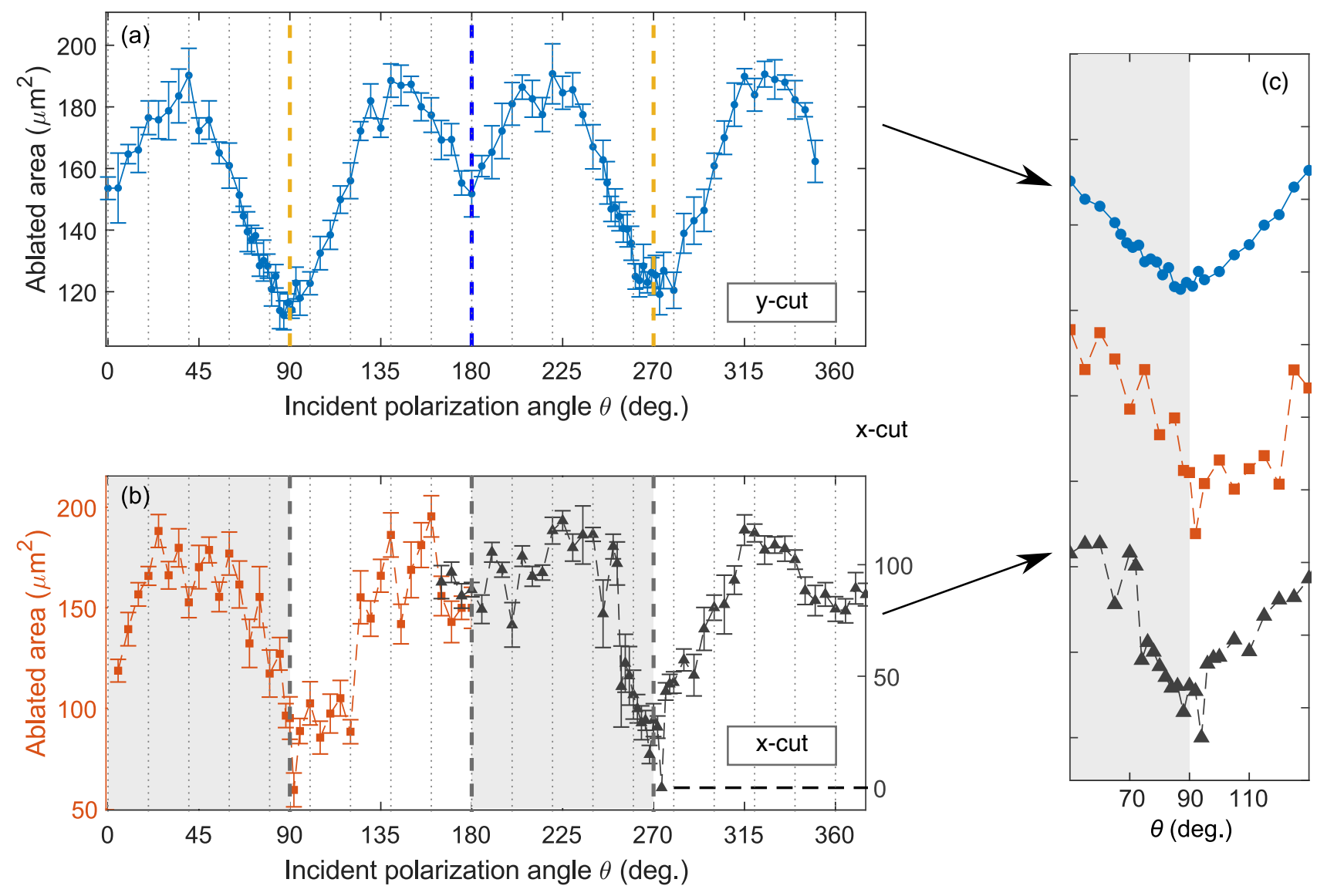

FIG. 2. Single-shot ablated area of $\mathrm{LiNbO}_{3}$ as a function of the incident laser polarization angle $\theta$. Each data point is averaged over 10 measurements and the standard deviation is shown. (a) The blue curve displayed from 0 to $355^{\circ}$ corresponds to the $y$ cut ablated at an intensity of $2.3 \times 10^{13} \mathrm{~W} / \mathrm{cm}^{2}$. Each half of the angular scan on either side of the central dashed blue line at $180^{\circ}$ exhibits the same polarization dependence. Also, a symmetric distribution is observed on both sides of the minima, which are identified by the dashed yellow lines at $90^{\circ}$ and $270^{\circ}$. The ablated area is maximal at $50^{\circ}$ apart from each minimum. (b) Orange squares show the results for an $x$-cut crystal ablated at the same laser intensity. An asymmetric structure is observed in the region where the ablated area is the smallest, characterized by a sharp decrease on the left-hand side (shaded area) and a smooth rise on the right-hand side. The black triangles from $165^{\circ}$ to $375^{\circ}$ show that this asymmetric feature is reproduced at a lower intensity $\left(1.9 \times 10^{13} \mathrm{~W} / \mathrm{cm}^{2}\right)$. In this case, ablation is completely suppressed at $\theta=274^{\circ}$ and no ablated area was observed. (c) Inset comparing each curve in the region where ablation is minimal.

has been shifted by $180^{\circ}$ and is displayed from $165^{\circ}$ to $375^{\circ}$. Noteworthily, the polarization dependence at this reduced intensity allows us to conveniently switch between operation above and operation below the ablation threshold. Indeed, it was possible to completely prevent ablation for the angle $\theta=274^{\circ}$.

For all measurements presented in Fig. 2, it is worth noting the significant variation of the ablated area provided by LIA and, thus, the very high measurement contrast. Depending on the experimental intensity, the observed contrast ranges from $41 \%$ ( $y$ cut, high intensity) to $100 \%$ ( $x$ cut, low intensity) with respect to the maximal value. Such variations have not been demonstrated with all-optical techniques, where the typical contrast is in the range of a few percent $[12,15]$.

\section{B. Crystal structure and symmetry}

The first element to explain the difference in angular response between the $x$ cut and the $y$ cut is found by looking at the symmetry of the crystal. $\mathrm{LiNbO}_{3}$ belongs to the hexagonal crystal family and is described by a trigonal system with a $C 3 v$ point group (space group $R 3 c$ ). A two-dimensional representation (top view) of the hexagonal structure is shown in Fig. 3(a). The trigonal system is characterized by a threefold rotation axis and the symmetry elements of the $C 3 v$ point group include three mirror planes that are located $60^{\circ}$ from each other, identified by dashed black and blue lines. In our work, the definition of axes follows the same standard convention as in $[18,19]$, thus the $y$ axis (blue arrow) in Fig. 3(b) lies in a plane of mirror symmetry, which is not the case for the $x$ axis (red arrow). For this reason, the left- and right-hand sides of the angular scan for the $y$ cut [Fig. 2(a)] show symmetric behavior with respect to $180^{\circ}$ since the sample surface intersects perpendicularly a mirror plane. Furthermore, Figs. 3(c) and 3(d) display a front view of the hexagonal structure of $\mathrm{LiNbO}_{3}$ for each crystal cut. For the $y$ cut [Fig. 3(d)], in addition to the left-right symmetry (dashed blue line) discussed above, the horizontal dashed yellow line allows us to visualize an up-down reflection symmetry for the niobium atoms that is absent for the $x$ cut. As described 

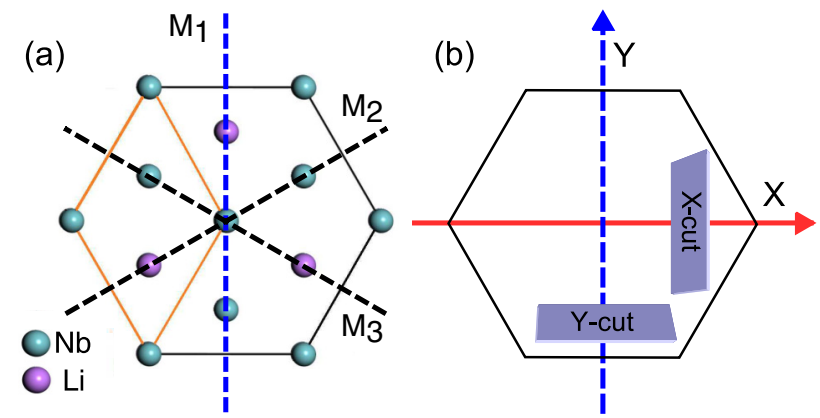

(c)

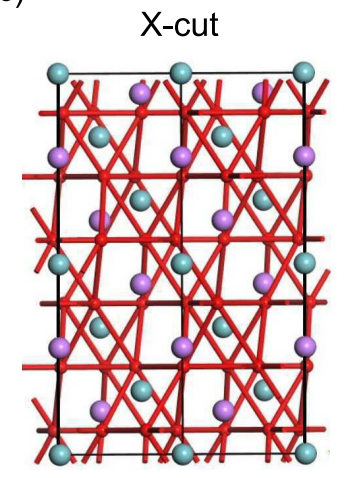

(d)

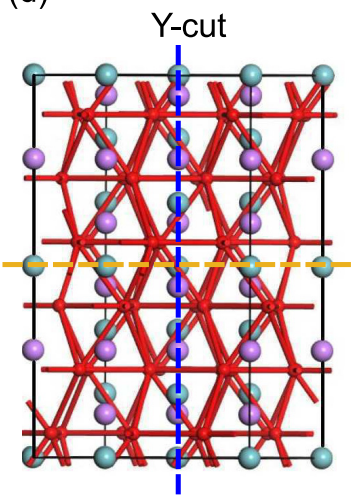

FIG. 3. (a) Top view of the hexagonal structure of $\mathrm{LiNbO}_{3}$. The edges of the conventional hexagonal unit cell are shown in orange and the dashed lines $\left(\mathrm{M}_{1}-\mathrm{M}_{3}\right)$ correspond to the characteristic mirror planes of the $C 3 v$ point group. (b) Illustration of the $x$ and $y$ cuts relative to the hexagonal structure. The polished surface of the $y$-cut sample intersects perpendicularly a mirror plane that is absent for the $x$ cut. (c), (d) Front view of the three-dimensional structure for each cut. For the $y$ cut, the atomic distribution displays a left-right symmetry (dashed blue line), as well as an up-down symmetry for the niobium atoms. This up-down symmetry, which is not present in the $x$ cut, is displayed as the horizontal dashed yellow line as a guide for the eye to compare both cuts. The dashed blue lines in (a), (b), and (d) correspond to the mirror plane shown in Fig. 2(a).

in the paragraph below, these $\mathrm{Nb}$ atoms being one of the main contributors to the crystal band structure, their spatial distribution within the unit cell also dictates the polarization dependence on the ionization of the material. For this reason symmetric behavior with respect to $90^{\circ}\left(270^{\circ}\right)$ is shown in Fig. 2(a), which is absent for the $x$ cut in Fig. 2(b).

\section{Density of states}

Additional insight about the role of the structure of $\mathrm{LiNbO}_{3}$ in the angular dependence of strong-field ionization can be gained from the calculated partial and total density of states (DOS) of the material shown in Fig. 4. Below the Fermi energy (vertical dashed pink line), the oxygen $2 p$ orbitals [Fig. 4(a)] define the valence band of the crystal, while the $4 d$ orbitals of the niobium atoms [Fig. 4(b)] define the conduction band above this level. The lithium atoms [Fig. 4(c)] do not contribute to any of the bands. The total DOS [Fig. 4(d)] that determines the band structure of the material is therefore defined by the $\mathrm{Nb}-\mathrm{O}$ bonds, in agreement with previous works $[20,21]$. The orbitals of these bonds form the smallest

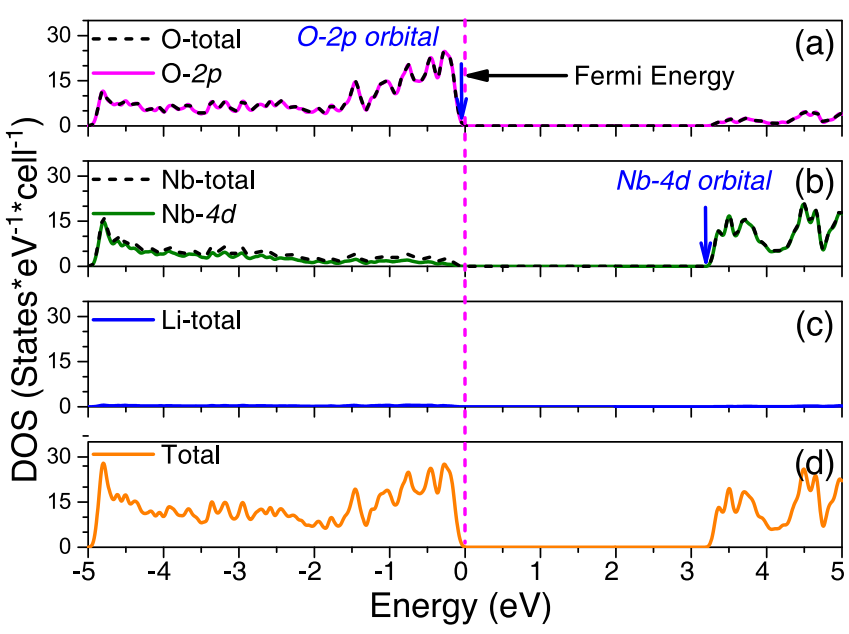

FIG. 4. Partial and total density of states (DOS) of $\mathrm{LiNbO}_{3}$ with the Fermi energy $(0 \mathrm{eV})$ identified by the dashed vertical line. (a) The valence band below the Fermi energy is almost exclusively composed of the oxygen $2 p$ orbitals of the crystal structure. (b) The conduction band above the Fermi energy is mainly defined by the niobium $4 d$ orbitals. (c) The contribution of lithium atoms is negligible. (d) Each curve is compared to this total DOS.

bandgap, where maximal ionization is expected because of the exponential dependence of tunneling on the bandgap energy. Figures 5(a) and 5(b), respectively, display the hexagonal unit cell of $\mathrm{LiNbO}_{3}$ and the corresponding charge density for the $\mathrm{Nb}-\mathrm{O}$ bonds along the $y$-cut surface. The relative angle for each bond with respect to the $c$ axis of the crystal is identified. From the DOS analysis, these orientations are subject to additional damages upon laser-induced ablation. Notably, in Fig. 5(b) two bonds, at $135^{\circ}$ (bond 1) and $222^{\circ}$ (bond 3), are quasiparallel to those laser polarization orientations where ablation is most efficient in Fig. 2(a), (a)

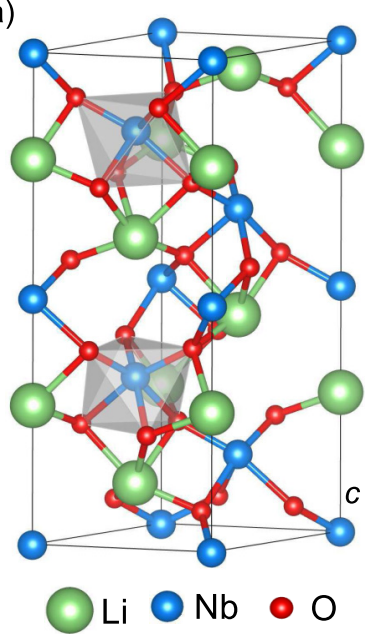

(b)
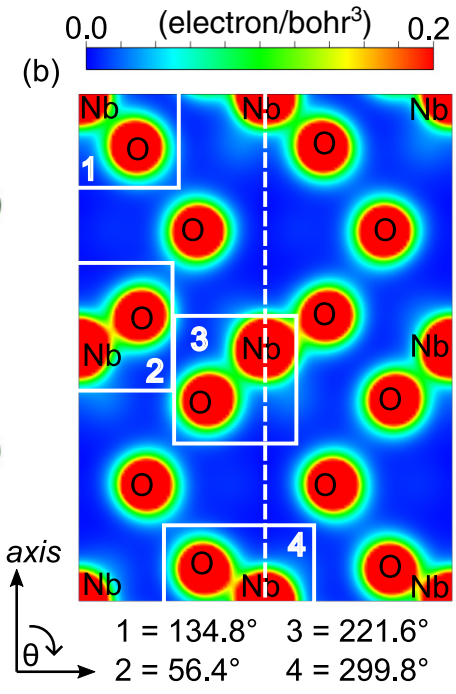

FIG. 5. Nb-O bond orientations in $\mathrm{LiNbO}_{3}$. (a) Hexagonal unit cell. (b) Cut of the calculated charge density along the $y$-cut surface. The relative angles between each $\mathrm{Nb}-\mathrm{O}$ bond and the $c$ axis of the crystal are shown. 
at $140^{\circ}$ and $220^{\circ}$, respectively. Because the experiment is not sensitive to the direction of the bond vectors but only to their orientation, these angles are also equivalent to $40^{\circ}$ and $320^{\circ}$, where an enhanced ablation is observed as well. The experimental results hence reveal with high contrast the main chemical bonds contributing to the ionization of the material.

\section{Semiconductor Bloch equations}

To obtain a microscopic description of our angle-resolved measurements, we modeled the strong-field ionization of the material. We solved the semiconductor Bloch equations applied to a two-band model for $\mathrm{LiNbO}_{3}$ and evaluated the conduction-band population resulting from the interaction with the strong laser field for different orientations of the incident polarization. The energy bands shown in Fig. 1(b), the charge density in Fig. 5, and the dipole matrix elements were obtained from first-principles density functional theory (DFT) calculations with the Vienna $a b$ initio Simulation Package [22]. The local density approximation was employed to describe the exchange-correlation functional [23]. The full potential projector-augmented-wave method was used with a plane-wave cutoff of $500 \mathrm{eV}$ [24]. In order to perform calculations for the converged wave function and band structure, the Brillouin zone of the unit cell was sampled with a $24 \times$ $24 \times 8$ Monkhorst-Pack $k$ grid for electronic self-consistent calculations. The resulting energy bands and dipole matrix elements were introduced into the semiconductor Bloch equations $[25,26]$.

Figure 6(a) shows how the conduction-band population varies as a function of the laser polarization angle for both the $x$ cut (black triangles) and the $y$ cut (blue circles). The $y$ cut shows symmetry with respect to a minimum centered at $90^{\circ}$, while for the $x$ cut, an asymmetry in the range of $75^{\circ}-110^{\circ}$ is observed (see inset). This agrees with the experimental measurements in Fig. 2 and the crystallography analysis in Fig. 3, confirming that the orientation dependence of the ablated area originates from the angle-dependent tunnel ionization. To further test the robustness of these observations, we performed additional calculations by varying the bandgap energy and dephasing time for the interband polarization. In all cases, the characteristic asymmetry for the $x$ cut is preserved. In fact, for each crystal cut the variation of the conduction-band population follows the evolution of the band structure for the different crystal orientations. This is shown in the Supplemental Material [25].

Furthermore, Fig. 6(b) displays the orientation-dependent reciprocal of the bandgap energy $1 / E_{g}$. Note that this value is averaged over all the $k$ points of the Brillouin zone rather than evaluated at the single gamma $(\Gamma)$ point since, in the latter case, the bandgap energy is independent of the crystal orientation. The symmetry/asymmetry shown in the inset highlights the importance of considering the entire bands in the tunnel ionization of the material.

\section{CONCLUSIONS}

In this work, we have characterized the laser polarization dependence of LIA for $x$-cut and $y$-cut lithium niobate,
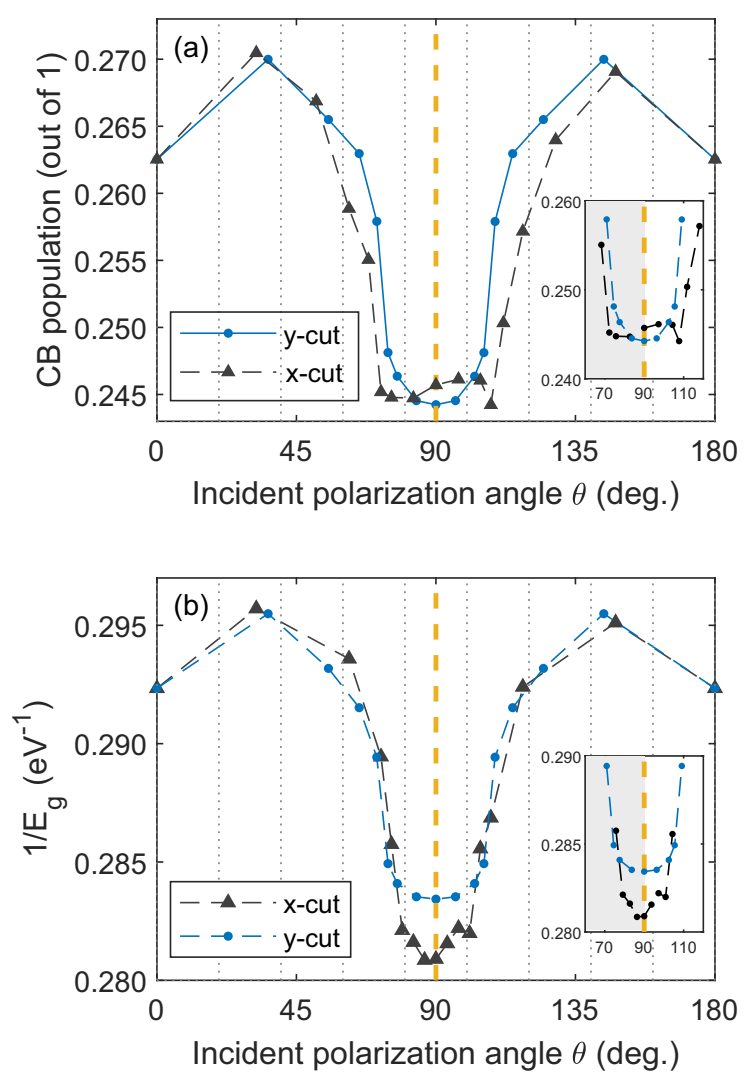

FIG. 6. (a) Calculated conduction-band (CB) population for $x$ and $y$-cut crystals as a function of the laser polarization angle $\theta$. (b) The corresponding reciprocal of the $k$-point-averaged bandgap energy. Insets: The region in the vicinity of the minimum where the symmetry/asymmetry is observed.

providing a macroscopic observable with a very high contrast compared to conventional all-optical techniques. By rotating linearly polarized $1800-\mathrm{nm}$ femtosecond laser pulses, we demonstrated that it is possible to image the mirror symmetries of the crystal along the plane ablated by the laser field. Moreover, the laser polarization angles where LIA is most efficient and thus leads to the largest ablated area were attributed to those angular regions containing the $\mathrm{Nb}-\mathrm{O}$ bonds of the structure. Through their charge density, the spatial distribution of these bonds dictates the symmetry features in the angle-resolved measurements. These characteristic features of the laser polarization dependence on LIA were reproduced for each crystal cut by solving the semiconductor Bloch equations for $\mathrm{LiNbO}_{3}$. In the typical two-band model employed the bands are frozen. However, it has recently been demonstrated that the high intensity of the laser field can also affect the temporal evolution of the band structure during ionization [28]. In a more advanced model, it would be interesting to consider this effect.

Finally, laser micromachining based on LIA is a privileged technique for high-precision material processing. As $\mathrm{LiNbO}_{3}$ is extensively used in optical devices that require the sample to be prepared using this technique [6], our results will contribute to reaching a better understanding and control over the writing procedure of this material. 


\section{ACKNOWLEDGMENTS}

The authors acknowledge the financial support from the Canada Foundation for Innovation (CFI), the Natural Sciences and Engineering Research Council of Canada (NSERC), the Fonds de recherche du Québec-Nature et technologies (FRQNT), PROMPT, and the National Natural Science Foundation of China (NSFC) (Grants No. 91850121, No. 11674363, No. 11561121002). V.W. was supported by the Vanier Canada Graduate Scholarship (Vanier CGS) program.
[1] K. Sugioka and Y. Cheng, Light: Sci. Appl. 3, e149 (2014).

[2] K. Sugioka, J. Xu, D. Wu, Y. Hanada, Z. Wang, Y. Cheng, and K. Midorikawa, Lab Chip 14, 3447 (2014).

[3] R. R. Gattass and E. Mazur, Nat. Photon. 2, 219 (2008).

[4] D. Brüske, S. Suntsov, C. E. Rüter, and D. Kip, Opt. Express 25, 29374 (2017).

[5] D. Brüske, S. Suntsov, C. E. Rüter, and D. Kip, Opt. Express 27, 8884 (2019).

[6] L. Li, W. Nie, Z. Li, Q. Lu, C. Romero, J. R. Vázquez De Aldana, and F. Chen, Sci. Rep. 7, 1 (2017).

[7] M. Lenzner, J. Krüger, W. Kautek, and F. Krausz, Appl. Phys. A: Mater. Sci. Process. 68, 369 (1999).

[8] V. Wanie, T. Shao, P. Lassonde, H. Ibrahim, J. Deschamps, J.-Q. Liu, F. A. Vargas, F. Vidal, A. Ruediger, F. Calegari, X.-B. Bian, and F. Légaré, Phys. Rev. B 101, 184103 (2020).

[9] C. B. Schaffer, A. Brodeur, and E. Mazur, Meas. Sci. Technol. 12, 1784 (2001).

[10] A. C. Tien, S. Backus, H. Kapteyn, M. Murnane, and G. Mourou, Phys. Rev. Lett. 82, 3883 (1999).

[11] J. B. Ashcom, R. R. Gattass, C. B. Schaffer, and E. Mazur, J. Opt. Soc. Am. B 23, 2317 (2006).

[12] M. Gertsvolf, H. Jean-Ruel, P. P. Rajeev, D. D. Klug, D. M. Rayner, and P. B. Corkum, Phys. Rev. Lett. 101, 243001 (2008).

[13] X. Li, W. Rong, L. Jiang, K. Zhang, C. Li, Q. Cao, G. Zhang, and Y. Lu, Opt. Express 22, 30170 (2014).

[14] F. Seitz, Phys. Rev 76, 1376 (1949).
[15] S. M. Golin, S. E. Kirkwood, D. D. Klug, D. M. Villeneuve, D. M. Rayner, C. A. Herrero, and P. B. Corkum, J. Phys. B 47, 204025 (2014).

[16] A. Dhar and A. Mansingh, J. Appl. Phys. 68, 5804 (1990).

[17] X. Liu, D. Du, and G. Mourou, IEEE J. Quantum Electron. 33, 1706 (1997).

[18] R. S. Weis and T. K. Gaylord, Appl. Phys. A 37, 191 (1985).

[19] S. Sanna and W. G. Schmidt, Phys. Rev. B 81, 214116 (2010).

[20] A. Riefer, S. Sanna, A. Schindlmayr, and W. G. Schmidt, Phys. Rev. B 87, 195208 (2013).

[21] R. Husin, F. W. Badrudin, M. F. Taib, and M. Z. Yahya, Mater. Res. Express 6, 114002 (2019).

[22] G. Kresse and J. Hafner, Phys. Rev. B 47, 558 (1993).

[23] W. Kohn and L. Sham, Phys. Rev. 140, A1133 (1965).

[24] P. E. Blöchl, Phys. Rev. B 50, 17953 (1994).

[25] See Supplemental Material at http://link.aps.org/supplemental/ 10.1103/PhysRevB.101.214311 for details on the theoretical model as well as complementary simulations. In particular, see Fig. S3 and Ref. [27] therein.

[26] G. Vampa, C. R. McDonald, G. Orlando, P. B. Corkum, and T. Brabec, Phys. Rev. B 91, 064302 (2015).

[27] G.-C. Wang and T.-M. Lu, RHEED Transmission Mode and Pole Figures (Springer, New York, 2014).

[28] C. R. McDonald, G. Vampa, P. B. Corkum, and T. Brabec, Phys. Rev. Lett. 118, 173601 (2017). 